\title{
A Defence of the Principle of Information Closure against the Sceptical Objection
}

\begin{abstract}
The topic of this paper may be introduced by fast zooming in and out of the philosophy of information. In recent years, philosophical interest in the nature of information has been increasing steadily. This has led to a focus on semantic information, and then on the logic of being informed, which has attracted analyses concentrating both on the statal sense in which $S$ holds the information that $p$ (this is what I mean by logic of being informed in the rest of this article) and on the actional sense in which $S$ becomes informed that $p$. One of the consequences of the logic debate has been a renewed epistemological interest in the principle of information closure (henceforth PIC), which finally has motivated a revival of a sceptical objection against its tenability first made popular by Dretske. This is the topic of the paper, in which I seek to defend PIC against the sceptical objection. If I am successful, this means - and we are now zooming out - that the plausibility of PIC is not undermined by the sceptical objection, and therefore that a major epistemological argument against the formalization of the logic of being informed based on the axiom of distribution in modal logic is removed. But since the axiom of distribution discriminates between normal and non-normal modal logics, this means that a potentially good reason to look for a formalization of the logic of being informed among the non-normal modal logics, which reject the axiom, is also removed. And this in turn means that a formalization of the logic of being informed in terms of the normal modal logic B (also known as KTB) is still very plausible, at least insofar as this specific obstacle is concerned. In short, I shall argue that the sceptical objection against PIC fails, so it is not a good reason to abandon the normal modal logic $\mathbf{B}$ as a good formalization of the logic of being informed.
\end{abstract}

\section{INTRODUCTION}

The topic of this article may be introduced by fast zooming in and out of the philosophy of information. ${ }^{1}$ In recent years, philosophical interest in the nature

$1 \quad$ See (Floridi 2011b). 
of information has been increasing steadily. ${ }^{2}$ This has led to a focus on semantic information, ${ }^{3}$ and then on the logic of being informed, ${ }^{4}$ which has attracted analyses concentrating both on the statal $^{5}$ sense in which $S$ holds the information that $p$ (this is what I mean by "logic of being informed" in the rest of this article) and on the actional sense in which $S$ becomes informed that $p$. One of the consequences of the logic debate has been a renewed epistemological interest in the principle of information closure (henceforth PIC), which finally has motivated a revival of a sceptical objection against its tenability. Dretske and Nozick (Dretske 1981, 1999, 2006; Nozick 1981) found the objection convincing and their support made it popular. The topic of this article is not a commentary on Dretske's position and the debate that it has generated, ${ }^{6}$ but rather a defence of PIC against the sceptical (or, rather, scepticism-based) objection. If I am successful, this means - and we are now zooming out - that the plausibility of PIC is not undermined by the sceptical objection. But since PIC is logically equivalent to the axiom of distribution, this is equivalent to showing that a major epistemological argument against the formalization of the logic of being informed, based on the axiom of distribution in modal logic, is removed. And since the axiom of distribution discriminates between normal and non-normal modal logics, this means that a potentially good reason to look for a formalization of the logic of being informed among the non-normal modal logics, ${ }^{7}$ which reject the axiom, is also removed. And this finally means that a formalization of the logic of being informed, in terms of the normal modal logic $\mathbf{B}$, is still plausible, at least insofar as this specific obstacle is concerned. In short, I shall argue that the sceptical objection against PIC fails, so the sceptical objection is not a good reason to abandon the normal modal logic $\mathbf{B}$ as a good formalization of the logic of being informed.

2 For an early overviews see (Floridi 2004).

3 At least since (Dretske 1981), see now (Dretske 1999). For an introduction see (Floridi 2011a).

4 See (Floridi 2006), revised as chapter 10 of (Floridi 2011b).

5 The statal condition of being informed is that enjoyed by $S$ once $S$ has acquired the information (actional state of being informed) that $p$. It is the sense in which a witness, for example, is informed (holds the information) that the suspect was with her at the time when the crime was committed. The distinction is standard among grammarians, who speak of passive verbal forms or states as "statal" (e.g. "the door was shut (state) when I last checked it") or "actional" (e.g. "but I don't know when the door was shut (act)").

6 On the debate see (White 1991), (Jäger 2004), (Baumann 2006), (Luper 2006), (Shackel 2006), (Dretske 2006). At the time of writing, the most recent contribution is (Adams et al.), which defends Dretske's position. In two recent articles, Genia Schoenbaumsfeld (Schoenbaumsfeld submitted-a, submitted-b) has defended the principle of epistemic closure from a Wittgensteinian perspective that converges with some of the conclusions reached in the following pages. I am grateful to her for sharing her research.

7 The analysis of the logic of being informed in terms of a non-normal modal logic is developed by (Allo 2011). 
The paper has the following structure. In Section 2, I formulate PIC against the background provided by the principle of epistemic closure (PEC). There I argue that a satisfactory formulation of PIC is in terms of the straight principle of information closure. In Section 3, I formulate the sceptical objection against PIC. In a nutshell, this is a modus tollens that holds that PIC is too good to be true: if PIC were acceptable, it would work as a refutation of radical scepticism, yet this violates a more general and widely accepted principle, according to which no amount of factual information can actually answer sceptical questions, so PIC must be rejected. In Section 4, I show that, although the argument is convincing, it mis-allocates the blame: it is not PIC that needs to be abandoned, but the assumption that one might be allowed to start with an uncontroversial piece of factual information, which then provides the input for the correct application of PIC, thus leading to the sceptical refutation. It follows that the sceptical objection does not undermine the tenability of PIC. There might be other good reasons to challenge information closure, but the "too good to be true" argument is not one of them. In Section 5, I consider a potential counter-argument, based on a different formulation of PIC in the context of empirical information processing and show that this too is ineffectual. In the conclusion, I indicate how the acceptance or rejection of PIC determines the choice of normal or non-normal modal logics that best model epistemic and information logics and remind the reader that the removal of the sceptical argument leaves open the choice of a normal modal logic.

\section{The formulation of the Principle of Information Closure}

Formulating the principle of closure in informational terms is not as straightforward as it might seem. This because PIC is often assumed, at least implicitly, to be a simplified version of the principle of epistemic closure (PEC), and there is quite a large variety of alternative formulations of the latter, each presenting some interesting if subtle mutations. ${ }^{8}$ Luckily, the informational translation makes our task less daunting because information is a more impoverished concept than that of knowledge and the ensuing minimalism does help to unclutter our conceptual space. Let us see how.

Initially, it might seem that the best way to formulate PIC would be to use the formulation of PEC under known entailment as a template, namely:

K If, while knowing that $p, S$ believes that $q$ because $S$ knows that $p$ entails $q$, then $S$ knows that $q$.

$\mathrm{K}$ looks like a good starting point because it includes, as an explicit requirement, the fact that $S$ holds (epistemically, doxastically or, in our case, informationally)

8 The interested reader is referred to the excellent review in (Luper 2010). In this article I use $\mathrm{K}$ and SP in the way in which they are used in the epistemological literature rather than in modal logic one (see below). 
not only that $p$ but also that $p$ entails $q$. As we shall see presently, this is an advantage, because it enables us to avoid a whole set of distracting issues, based on the contingent or idiosyncratic unavailability of the entailment to a particular $S$. The fact that Peter might fail to hold the information that Paris is in Europe, while holding the information that Paris is in France, because Peter misses the information that France is in Europe and therefore fails to hold that if Paris is in France then Paris is in Europe, might be relevant in other contexts, e.g. to check how well informed Peter is about European geography, but not here. As it will become clearer in the next two sections, the argument using the sceptical objection attacks PIC not because people have informational or cognitive limits - of course we all do, since we may be distracted, lack a crucial piece of information, be incapable to see what follows from the information that we do hold, run out of time to perform the required logical steps, etc. - but because, if we concede information about both premises, we seem to be able to refute the sceptic, and this, for reasons to be discussed, is alleged to be unacceptable.

The good news is therefore that the requirement of known entailment is a positive feature in $\mathrm{K}$. The bad news is that, despite this, the informational translation of $\mathrm{K}$ does not work. Suppose we simplify our task and avoid any reference to beliefs or knowledge. The rationale for this is that we are seeking to formulate a principle of information closure with a broader basis of applicability: it should work for human and artificial agents - including computers that may be able to hold information physically - and hybrid agents, like banks or online services, which might hold information in their files, or in the memories of their employees. Neither artificial nor hybrid agents can be said to believe or know that $p$ nonmetaphorically, for they lack the required mental states or propositional attitudes. In this case, $\mathrm{K}$ becomes the principle of known information closure:

PKIC If, while holding the information that $p, S$ holds the information that $q$ because $S$ holds the information that $p$ entails $q$, then $S$ holds the information that $q$.

Clearly, PKIC will not do, for it just trivialises the principle into a verbose repetition. If $S$ holds the information that $q$ then $S$ holds the information that $q$ : uncontroversial but also useless. Although it would be interesting to investigate why the informational translation deprives $\mathrm{K}$ of its conceptual value, this would go well beyond the scope of this article, so let us not get side-tracked. More constructively, let us keep the known entailment clause in $\mathrm{K}$, which we have seen to be a valuable feature, and use it to modify another version of PEC, known as the straight principle of epistemic closure. This states that:

SP If $S$ knows that $p$, and $p$ entails $q$, then $S$ knows that $q$.

The modification, translated in informational terms, gives us:

SPIC If $S$ holds the information that $p$, and $S$ holds the information that $p$ entails $q$, then $S$ holds the information that $q$. 
SPIC treats $p$ entails $q$ as another piece of information held by $S$, as required by the known entailment feature. This avoids contingent or idiosyncratic distractions, as we have seen above in the "French" example with Peter.

Following (Floridi 2006), we obtain what may be called the canonical principle of information closure:

$$
\text { PIC }(I p \wedge I(p \rightarrow q)) \rightarrow I q
$$

PIC is not trivial, or at least not in the sense in which PKIC above is. It also appears to deliver exactly what we need in order to analyse the sceptical objection informationally.

The last step concerns how we handle the entailment with the wider scope occurring in PIC. Mind, I do not say interpret it, for this is another matter. In the rest of our analysis, I suggest we simplify our task by following the common assumption according to which both entailments are interpreted in terms of material implication. It is the main entailment in PIC that can be handled in several ways. I shall mention two first, for they provide a good introduction to a third one that seems preferable for our current purpose.

A modest proposal is to handle the entailment in terms of feasibility. $S$ could obtain the information that $q$, if only $S$ cares enough to extract it from the information that $p$ and the information that $p$ entails $q$, both of which are already in $S$ ' possession. Consider the following example. The bank holds the information that Peter, its chairman, is overpaid. As a matter of fact, the bank also holds the information (endorses the entailment) that, if its chairman is overpaid, then he does not qualify for an annual bonus. So the bank can (but might not) do something with the entailment. Peter might keep receiving his annual bonus for as long as the bank fails to use or indeed decides to disregard the information at its disposal to generate the information that Peter no longer qualifies and then act on it.

A slightly more ambitious proposal, which has its roots in work done by (Hintikka 1962), is to handle the entailment normatively: S should obtain the information that $q$. In our example, the bank should reach the conclusion that Peter no longer qualifies for an annual bonus; if it does not, that is a mistake, for which someone (e.g., an employer) or something (e.g., a department) may be reprimanded.

A further alternative, more interesting because it bypasses the limits of the previous two, is to handle the entailment as part of a sufficient procedure for information extraction (data mining): in order to obtain the information that $q$, it is sufficient for $S$ to hold the information that $p$ entails $q$ and the information that $p$. This third option captures the view that PIC works like an algorithm, with a rule, $I(p \rightarrow q)$, an input $I p$ and an output $I q$. It also leaves unspecified whether $S$ will, can or even should extract $q$. One way for the bank to obtain the information that Peter does not qualify for an annual bonus is to hold the information that, if he is overpaid, then he does not qualify for an annual bonus, and the information that Peter is overpaid. Handling the entailment as part of a sufficient procedure for 
information extraction means qualifying the information that $q$ as obtainable independently of further experience, evidence, or input, that is, it means showing that $q$ is obtainable without overstepping the boundaries of the available information base. This is just another way of saying that the information in question is obtainable a priori.

We now have a satisfactory formulation and interpretation of the principle of information closure. Let us look at the sceptical objection.

\section{THE SCEPTICAL OBJECTION}

The sceptical objection against PIC has been formulated and debated in several papers. Essentially, it is a modus tollens, which requires three steps. The first two are very simple. They consist in providing an interpretation of the information that $p$ and of the information that $q$ such that $p$ entails $q$. The reader is welcome to provide her own version. Here, I shall follow (Kerr and Pritchard forthcoming), and use:

$$
\begin{array}{ll}
p:= & S \text { is in Edinburgh } \\
q:= & S \text { is not a brain in a vat on Alpha Centauri [henceforth BiVoAC]. } \\
e:= & \text { If } S \text { is in Edinburgh then } S \text { is not a brain in a vat on Alpha Centauri } \\
& \text { [henceforth BiVoAC]. }
\end{array}
$$

As Kerr and Pritchard remark, referring to Dretske's rejection of PIC:

[...] on Dretske's view I can have an informational basis for believing that I am in Edinburgh but I can have no informational basis for believing that I am not a BIV [brain in a vat] on Alpha Centauri (a skeptical hypothesis which entails that I am not in Edinburgh), even whilst I know that if I am a BIV on Alpha Centauri then I am not in Edinburgh. It is for this reason that Dretske denies epistemic [information] closure.

The third step is the formulation and adoption of a negative thesis:

NT information alone cannot answer a sceptical doubt.

NT seems most plausible. It refers to factual information, and it is a standard assumption in the literature on scepticism, from Sextus Empiricus to Descartes to Wittgenstein. It is explicitly proposed by Dretske himself, shared by Kerr and Pritchard, and I agree with them: sceptical doubts of a Cartesian nature cannot be answered by piling up more or different kinds of factual information. One of the reasons for raising them is precisely because they block such possibility. We would have stopped discussing sceptical questions a long time ago if this were not the case.

We are now ready to formulate the sceptical objection against PIC thus:

i) if PIC, $p$ and $e$ 
ii) then $S$ can generate the information that $q$ a priori;

iii) but $q$ is sufficient for $S$ to answer the sceptical doubt (in the example, $S$ holds the information that $S$ is not a BiVoAC);

iv) and (iii) contradicts NT;

v) but NT seems unquestionable;

vi) so something is wrong with (i)-(iii): in a Cartesian scenario, $S$ would simply be unable to discriminate between being in Edinburgh or being a $\mathrm{BiVoAC}$, yet this is exactly what has just happened;

vii) but (iii) is correct;

viii) and the inference from (i) to (ii) is correct;

ix) and $e$ in (i) seems innocent;

x) so the troublemaker in (i) is PIC, which needs to be rejected.

It all sounds very convincing, but I am afraid PIC has been framed, and I hope you will agree with me, once I show you by whom.

\section{THE DEFENCE OF THE PRINCIPLE}

Admittedly, PIC looks like the only suspicious character in (i). However, consider more carefully what PIC really achieves, that is, look at $e$. The entailment certainly works, but does it provide any information that can answer the sceptical doubt? Not by itself. For $e$ works even if both $p$ and $q$ are false, of course. This is exactly as it should be, since valid deductions, like $e$, do not generate new information, a scandal (D'Agostino and Floridi 2009) that, for once, it is quite useful to expose. Not only factual information alone cannot answer a sceptical doubt, deductions alone can never answer a sceptical doubt, either. If $e$ did generate new information, we would have a bizarre case of synthetic a priori reasoning (recall the handling of the entailment as a sufficient procedure for information extraction), and this seems a straightforward reductio. The fact is that the only reason why we take $e$ to provide some anti-sceptical, factual information about $S^{\prime}$ actual location in space and time is because we also assume that $p$ in $e$ is true. Ex hypothesis, not only $S$ is actually in Edinburgh, but $S$ holds such information as well. So, if PIC works anti-sceptically, it is because $q$ works anti-sceptically, but this is the case because $e+p$ work anti-sceptically, but this is the case only if $p$ is true. Now, $p$ is true. Indeed, it should be true, and not just in the chosen example, but in general, or at least for Dretske and anyone else, including myself, who subscribes to the veridicality thesis, according to which $p$ qualifies as information only if $p$ is true. But then, it is really $p$ that works anti-sceptically. All the strength in the antisceptical interpretation of (i)-(iii) comes from the truth of $p$ as this is known to $S$, that is, it comes from assuming that $S$ is informed that $p$. This becomes obvious once we realise that no shrewd sceptic will ever concede $p$ to $S$ in the first place, because she knows that, if you concede $p$, then the sceptical challenge is over, as Descartes 
correctly argued. Informationally (but also epistemically), it never rains, it pours: you never have just a bit of information, if you have some you ipso facto have a lot more. Quine was right about this. Allow a crack in the sceptical dam and the informational flooding will soon be inevitable. In a more epistemological vocabulary, if you know something, you know a lot more than just that something. This is why, in the end, local or circumscribed scepticism is either critical thinking under disguise or must escalate into global scepticism of a classic kind, e.g. Pyrrhonian or Cartesian. The conclusion is that it is really the initial input surreptitiously provided by $p$ that is the real troublemaker. PIC is only following orders, as it were. For PIC only exchanges the higher informativeness of a true $p$ (where $S$ is located, in our example) into the lower informativeness of a true $q$ (where $S$ is not located, being located where he is). This is like exchanging a twenty pounds banknote into many one-dollar bills. It might look like you are richer, but of course you are just a bit poorer, in the real life analogy because of the exchange rate and the commission charged, and in the sceptical objection because you moved from a positive statement (where you actually are located) to a negative one (one of the infinite number of places where you are not, including places dear to the sceptic like vats in Alpha Centauri). If you do not want the effects of $q$, - if you think that it is rather suspicious to end up with so many dollars coming out of nowhere - do not blame PIC, just never concede $p$ in the first place - do not give away the initial British pounds to begin with, using the cash analogy.

It follows that the informational answer to the sceptical doubt, which we agreed was an impossibility, is provided not by $q$, but by $p$, and this disposes of the objection that PIC is untenable because factual information can never provide an answer to sceptical doubts. It never does because one may never be certain that one holds it (one cannot assume to be informed that $p$ ), not because, if one holds it, it does not.

It might be remarked that all this leaves the last word to the sceptic. I agree, it does, but it does only in this context, and this is harmless. PIC was never meant to provide an antisceptical argument in the first place. It was the alleged accusation that it did in a mistaken way that was the problem. So what happens next? If being in Edinburgh means that I may not be sure that I am there, then we are talking about a scenario in which no further empirical information, no matter how far-reaching, complex, sophisticated or strongly supported, will manage to eradicate once and for all such Cartesian doubt. I believe it is this the proper sense in which all the factual information in the world will never meet the sceptical challenge. For factual information is a matter of empirical facts, and sceptical doubts are based on logical possibilities that challenge the reliability of all such facts. So the no reference to empirical facts, or no offer of factual information can cure logically possible doubts. If you are really worried about being a butterfly that is dreaming to be a human being, showing you that you cannot fly will not work. Is this, then, finally a good reason to reject PIC? The answer is again in the negative. PIC was not guilty when we were assuming to have a foot in the door, a piece of 
factual information about how the world really is, namely $p$. It is still not guilty now that we are dealing with a web of information items that might turn out to be a complete fabrication. On the contrary, in the former case it is PIC that helps us to squeeze some (admittedly rather useless) further bits of information from $p$. In the latter case, it is still PIC (though of course not only PIC) that makes the coherence of the whole database of our information tight. But if PIC is to be retained in both cases, what needs to be discharged? Either nothing, if we are allowed a foot in the door, because this is already sufficient to defeat the sceptical challenge; or the value of absolute scepticism as a weapon of total information destruction, if all that it can ever mean is that the logically possible is empirically undefeatable. Once made fully explicit and clarified in detail, radical informational scepticism, with its fanciful scenarios of possible worlds, can be proved to be entirely redundant informationally (Floridi 2010), so it can be disregarded as harmless. Wondering whether we might be dreaming, or living in a Matrix, or might be butterflies who think they are humans, or might be characters in a sci-fi simulation created by some future civilization, and so forth, are interesting speculations that may be intellectually stimulating or simply amusing, but that make no significant difference whatsoever to the serious problem of how we acquire, manage, and refine our information about the world when in the world. The endless game of dealing with them can be left to scholastic philosophers dreaming of final refutations.

\section{An OBJECTION AGAINST THE DEFENCE AND A REPLY}

The reader might still be unconvinced. There might be a lingering doubt about the value of PIC. Such doubt may turn into an objection against the previous defence of PIC that can be formulated by adapting (Adams 2011), who, following Dretske, argues that we should reject information closure. Here it is.

As Adams notices, I too reject PIC in cases in which the kind of information processing in question is empirical, as when we see or hear that such and such is the case. As I acknowledged in the past:

Not all "cognitive" relations are distributive. "Knowing", "believing" and "being informed" are, as well as "remembering" and "recalling". This is why Plato is able to argue that a "mnemonic logic", which he seems to base on $K 4$, may replace $D L$ [Doxastic Logic] as a foundation for $E L$ [Epistemic Logic]. However, "seeing" and other experiential relations, for example, are not: if an agent a sees (in a non metaphorical sense) or hears or experiences or perceives that $p \rightarrow q$, it may still be false that, if a sees (hears etc.) $p$, then a also sees (hears etc.) q. (Floridi 2006, p. 441.)

Adams would like to see a more uniform approach and argues that I should simply reject PIC in all cases. I resist it, but we might not be at variance. Consider the following case. 
In the left pocket of your jacket you hold the information that, if it is Sunday, then the supermarket is closed. Your watch indicates that today is Sunday. Do you hold the information that the supermarket is closed today? The unexciting answer is maybe. Perhaps, as a matter of fact, you do not, so Adams (and Dretske with him) is right. You might fail to make the note in the pocket and the date on the watch "click." Nevertheless, I would like to argue that, as a matter of logic, you should, that is, in terms of feasibility, normativity or sufficient procedure for information extraction you did have all the information that the supermarket was closed. So much so that you will feel silly when you are in front of its closed doors and realise that, if you had been more careful, you had all the information necessary to save you the trip. You should have known better, as the phrase goes. Now, I take logic to be a prescriptive not a descriptive discipline. From this perspective, PIC seems to be perfectly fine. This means that the logical application of PIC to informational co-variance is correct. Suppose two systems $a$ and $b$ are coupled in such a way that $a$ 's being (of type, or in state) $F$ is correlated to $b$ being (of type, or in state) $G$, so that $F(a)$ carries (for the observer of $a$ ) the information that $G(b){ }^{9}$ An application of PIC in this case means that, if $F(a) \rightarrow G(b)$ qualifies as information and so does $F(a)$, then $G(b)$ qualifies as well. For example, if the low-battery indicator $(a)$ flashing $(F)$ indicates that the battery $(b)$ is flat $(G)$ qualifies as information, and if the battery indicator flashing also counts as information, then so does the battery being flat.

Still from the same perspective, one should not jump to the conclusion that PIC is always applicable to any empirical way of handling information. Consider the example above. This time you read the following e-mail, sent by the supermarket: "The shop will be closed every Sunday." You also read the date on your computer, which correctly indicates that today is Sunday. Have you read that the supermarket is closed today? Of course not, as we assume that there were no further messages. Should you have read that it was? Obviously not, for where was the text that you should have read? Should you have inferred that the supermarket was closed today? Surely, for that was the information that could easily be extracted from the two texts that you read. Again, imagine you are in a hurry and you have only two "time tokens", let us say two seconds. And suppose that reading each message takes one token each. Clearly you do not have the time to extract the information that the supermarket is closed. In more abstract terms, the agent may simply lack the resources to extract not just all (since this is trivially true) but even the relevant information that is logically extractable from the available database.

Adams is talking about the performance of actual players, I am talking about the rules of the game. If Adams's thesis is that PIC is at best only a matter of logic and certainly not an empirical fact, I am convinced.

9 Such co-variance principle has been at the core of the philosophy of information at least since its explicit formulation in (Dretske 1981). The version provide here is from (Floridi 2011b, p. 41), which is a slight modification of the version provided by (Barwise and Seligman 1997). 


\section{Conclusion: Information Closure AND the LOGIC OF BEING INFORMED}

In this article, I have sought to defend the principle of information closure (PIC) against a popular objection, namely that its assumption would lead to an implausible argument that would defeat radical scepticism. I have shown why such an objection is misdirected. The previous debate might seem to be of interest only to epistemologists or philosophers of information, but such impression would be mistaken. The acceptance or rejection of the principle of closure in epistemology or in the philosophy of information has a wider consequence, in terms of the kind of modal systems that then become available to model epistemic and information logics of different strengths. Quite surprisingly for a topic so well discussed and understood, it seems that such consequence has remained implicit so far, and yet, it is very straightforward. Let me explain. The axiom of distribution states that: ${ }^{10}$

$$
\text { AOD } \quad \square(\varphi \rightarrow \psi) \rightarrow(\square \varphi \rightarrow \square \psi)
$$

AOD discriminates between normal modal logics, to which the axiom applies, and non-normal ones, where the axiom does not apply. PIC is simply the counterpart of AOD in the philosophy of information. This is because PIC can be translated as $(\square \varphi \wedge \square(\varphi \rightarrow \psi)) \rightarrow \square \psi)$, and the latter is logically equivalent to $\square(\varphi \rightarrow \psi) \rightarrow(\square \varphi \rightarrow \square \psi)$, as a reformulation of both in an implication-free form easily shows. Indeed, AOD is the source of the debate on PEC in modal logic. The parallel is enlightening once it is realised that arguments against AOD in terms of logical omniscience have the same conceptual format as scepticism-based arguments against PIC discussed in above: they are both based on a "too good to be true" strategy.

The fact that PIC and AOD are two sides of the same coin means that the acceptance or rejection of PIC determines whether one is going to consider normal or non-normal modal logics as more suitable to capture all the features one wants to include in an epistemic or information logic. There are good reasons for choosing either option, but two points should now be clear. One is a matter of consistency: rejecting PIC means rejecting the option that epistemic or information logics are normal modal logics. Such rejection is perfectly reasonable and (Allo 2011), for example, offers an interesting analysis of a non-normal alternative. However, and this is the second point, the refutation of the "sceptical argument" against PIC means that one obstacle against a normal modal logic analysis of " $S$ is informed that $p$ " has been removed. And this, in turn, means that the argument in favour of the analysis of information logic in terms of the normal modal logic $\mathbf{B}$ remains unaffected in this respect.

10 See for example (Cocchiarella and Freund 2008; Hughes and Cresswell 1984). The axiom is also and perhaps better known as the $\mathrm{K}$ axiom, but such terminology would be confusing in this paper. A less popular name is deductive cogency axiom. 


\section{REFERENCES}

Adams, F., 2011, "Information and Knowledge À La Floridi”, in P. Allo (Ed.), Putting Information First: Luciano Floridi and the Philosophy of Information. Oxford: Wiley-Blackwell, pp. 84-96.

Adams, F., Barker, J., and Figurelli, J. (forthcoming), "Towards Closure on Closure", in: Synthese, pp. 1-18.

Allo, P., 2011, “The Logic of 'Being Informed' Revisited and Revised”, in: Philosophical Studies, 153, 3, pp. 417-434.

Barwise, J. and Seligman, J., 1997, Information Flow: The Logic of Distributed Systems. Cambridge: Cambridge University Press.

Baumann, P., 2006, “Information, Closure, and Knowledge: On Jäger's Objection to Dretske", in: Erkenntnis, 64, 3, pp. 403-408.

Cocchiarella, N. B. and Freund, M. A., 2008, Modal Logic: An Introduction to Its Syntax and Semantics. New York-Oxford: Oxford University Press.

D’Agostino, M. and Floridi, L., 2009, “The Enduring Scandal of Deduction. Is Propositional Logic Really Uninformative?”, in: Synthese, 167, 2, pp. 271315.

Dretske, F., 1981, Knowledge and the Flow of Information. Oxford: Blackwell.

Dretske, F., 1999, Knowledge and the Flow of Information. Stanford, CA: CSLI Publications.

Dretske, F., 2006, “Information and Closure”, in: Erkenntnis, 64, 3, pp. 409-413.

Floridi, L., 2004, The Blackwell Guide to the Philosophy of Computing and Information. Oxford: Blackwell.

Floridi, L., 2006, “The Logic of Being Informed”, in: Logique et Analyse, 49, 196, pp. 433-460.

Floridi, L., 2010, "Information, Possible Worlds, and the Cooptation of Scepticism”, in: Synthese 175, pp. 63-88.

Floridi, L., 2011a, "Semantic Conceptions of Information", in E. N. Zalta (Ed.), The Stanford Encyclopedia of Philosophy. Spring Edition.

Floridi, L., 2011b, The Philosophy of Information. Oxford: Oxford University Press.

Hintikka, J., 1962, Knowledge and Belief: An Introduction to the Logic of the Two Notions. Ithaca: Cornell University Press.

Hughes, G. E. and Cresswell, M. J., 1984, A Companion to Modal Logic. London: Methuen. 
Jäger, C., 2004, "Skepticism, Information, and Closure: Dretske's Theory of Knowledge", in: Erkenntnis 61, 2-3, pp. 187-201.

Kerr, E. T. and Pritchard, D. (forthcoming), "Skepticism and Information", in: H. Demir (Ed.), Luciano Floridi's Philosophy of Technology. New York: Springer.

Luper, S., 2006, "Dretske on Knowledge Closure", in: Australasian Journal of Philosophy 84, 3, pp. 379-394.

Luper, S., 2010, “The Epistemic Closure Principle”, in: E. N. Zalta (Ed.), Stanford Encyclopedia of Philosophy.

Nozick, R., 1981, Philosophical Explanations. Oxford: Clarendon Press.

Schoenbaumsfeld, G. (submitted-a), "Mcdowellian Neo-Mooreanism?"

Schoenbaumsfeld, G. (submitted-b), "Meaning and Conversational Impropriety in Sceptical Contexts".

Shackel, N., 2006, "Shutting Dretske's Door", in: Erkenntnis 64, 3, pp. 393-401.

White, J. L., 1991, "Knowledge and Deductive Closure", Synthese 86, 3, pp. 409423.

Department of Philosophy

University of Hertfordshire

de Havilland Campus

Hatfield, Hertfordshire AL10 9AB

UK

1.floridi@herts.ac.uk 